\title{
Properties of non-crystalline EuIG and DyIG obtained from Mössbauer and magnetization data ${ }^{\mathrm{a}}$
}

\author{
F. J. Litterst, J. Tejada, ${ }^{b}$ and G. M. Kalvius \\ Physik Department E15, Technische Universität München, D-8046 Garching, Federal Republic \\ of Germany
}

Non-crystalline (nc) EuIG and DyIG have been prepared by dc-sputtering. Mössbauer data on ${ }^{57} \mathrm{Fe}$,

${ }^{151} \mathrm{Eu}$ and ${ }^{16 \mathrm{i}} \mathrm{Dy}$ reveal sharp magnetic transitions at $62 \mathrm{~K}$ and $70 \mathrm{~K}$ for nc EuIG and DyIg, respectively. The ${ }^{57} \mathrm{Fe}$ hyperfine (hf) spectra consist of three superpositioned patterns for $\mathrm{Fe}^{3+}$ in tetrahedral and octahedral and for $\mathrm{Fe}^{2+}$ in tetrahedral oxygen coordination. The saturation hf fields at $4.2 \mathrm{~K}$ are reduced compared to the values of the corresponding crystalline materials. The induced hf field at ${ }^{151} \mathrm{Eu}$ is only $1 / 8$ of that for crystalline EuIG.

PACS numbers: $75.50 . \mathrm{Kj}, 76.80 .+\mathrm{y}, 81.15 . \mathrm{Cd}$

\section{INTRODUCTION}

The well-known dependence on preparation conditions of the microstructural polymorphism in nc networks is often also reflected in their magnetic properties. This is particular$1 \mathrm{y}$ evident for non-metallic nc materials where superexchange coupling dominates. The latter is very sensitive to slight variations in bond lengths and angles. For example, this counterplay of local structural and magnetic properties has successfully been investigated by Mössbauer spectroscopy on nc ferrous halides $1-3$. Here we report about the characterization of nc EuIG and DyIG with the help of Mössbauer and macroscopic magnetization measurements.

\section{EXPERIMENTAL}

NC EUIG and DyIG have been prepared by slow dc-sputtering ( $8 \mathrm{kVAr}+$ ) from crystalline garnet targets. The argon pressure was 10-5Torr. Both, the target and the mylar substrate were kept at $\backsim 80 \mathrm{~K}$. Samples of thicknesses between some 103 up to 105 here obtained with deposition times ranging from 10 to 100 hours. Mössbauer absorption studies on $57 \mathrm{Fe}$ and $151 \mathrm{Eu}$ were carried out in the temperature range between 4.2 and $300 \mathrm{~K}$. Additiona measurements with 161 Dy are presently 1 imited to the range above $70 \mathrm{~K}$. Macroscopic magnetization data between 4.2 and $200 \mathrm{~K}$ were obtained with a vibrating sample magnetometer. $x$-ray characterization of the samples was done at room temperature,

\section{RESULTS}

The sputtered garnet samples are $x$-ray amorphous. Crystallization can be achieved by heating in air to $800-1000 \mathrm{~K}$. 57Fe Mössbauer spectra of nc EuIG and DyIG taken at $300 \mathrm{~K}$ in the paramagnetic regime are shown in Fig.l. In Fig. 2 we show spectra in the magnetically ordered state at $4.2 \mathrm{~K}$. The high temperature spectra can be interpreted by a superposition of three quadrupole patterns reflecting differ-

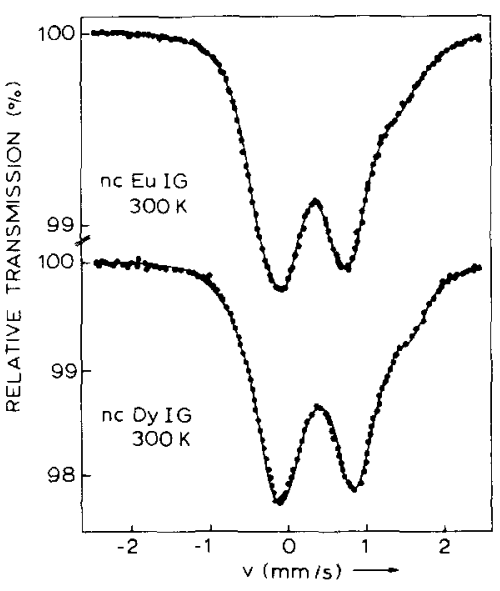

Fig. 1: ${ }^{57} \mathrm{Fe}$ absorption spectra of $n \mathrm{C}$ EUIG and DyIG at $300 \mathrm{~K}$. (source $30 \mathrm{mCi} 57 \mathrm{CoRh}$, eff. absorber thickness 0.10 and $0.15 \mathrm{mg}$

ent Fe sites. The values of their spectral parameters are given in Table I. One can tentatively identify tetrahedral and octahedral $\mathrm{Fe}^{3+}$ sites. The hf parameters of the third site are close to those known for tetrahedral $\mathrm{Fe}^{2+}$ (e.g. in silica garnets 6 ). The relative intensities of the three subspectra were corrected for different Lamb-Mössbauer factors (as estimated from their temperature dependence between 80 and $300 \mathrm{~K}$ ) in order to obtain the occupancy of the three sites (Table I).

For an accurate determination of the magnetic ordering temperature $\mathrm{T}_{\mathrm{m}}$ we performed thermal scanning 7 for the resonances of $57 \mathrm{Fe}$, $151 \mathrm{Eu}$ resp. $161 \mathrm{Dy}$. We found $\mathrm{T}_{m}=62 . \pm 0.5 \mathrm{~K}$ for nc EuIG and $70 . \pm 0.5 \mathrm{~K}$ for nc DyIG. The $57 \mathrm{Fe}$ magnetic hf patterns below $T_{m}$ were fitted by a superposition of three sets for 6-1ine effective field patterns. Their isomer shifts and the relative intensities were fixed to the 


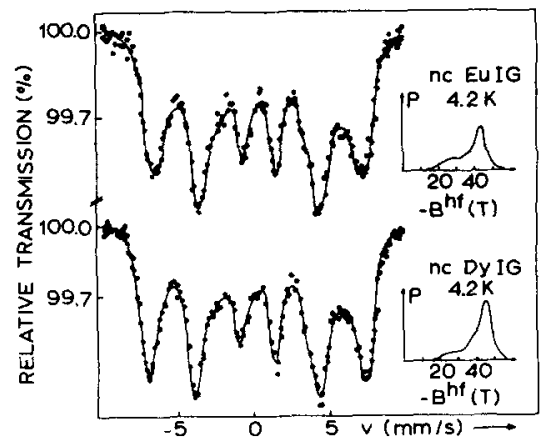

Fig.2: ${ }^{57} \mathrm{Fe}$ absorption spectra of nC EuIG and DyIG at $4.2 \mathrm{~K}$, the inserts give the probability distribution of $B^{h f}$.

values valid above $T_{m}$ (Table I). No variation in spectral shape was found by changing the angle between the direction of the $\gamma$-rays and the plane of the absorber substrate. This indicates the absence of a preferred orientation of the $\mathrm{Fe}$ moments. The distribution of the magnetic hf fields can be satisfactorily described by a sum of three Lorentzians. The effective hf fields Bhf for the three Fe sites and the relative widths of the distributions at $4.2 \mathrm{~K}$ are also summarized in Table I. In Fig. 3 the variation of $B$ hf with temperature is plotted for nc DyIG. The shape of the distribution function of $B$ hf (as shown in the inserts in Fig.2 for $4.2 \mathrm{~K}$ ) is qualitatively maintained up to $\mathrm{T}_{\mathrm{m}}$.

The Eu 34 ions experience on $1 y$ an induced hf magnetic field. A value of $8 T$ was found at $4.2 \mathrm{~K}$ which is about $1 / 8$ of the value for crystalline EuIG 8 . The quadrupole interaction above $T_{m}$ corresponds to $e^{2} q Q \mathrm{gd} \approx 130 \mathrm{MHz}$ for
$151 \mathrm{Eu}^{3+}$ in nC EuIG and $\approx 1600 \mathrm{MHz}$ for $161 \mathrm{Dy}^{3+}$ in nc DyIG.

From macroscopic magnetization measurements we find roughly a curie-Weiss behaviour above $90 \mathrm{~K}$. The extrapolated Curie-Weiss temperatures are $-220 \mathrm{~K}$ and $-120 \mathrm{~K}$ for $\mathrm{nC} E \mathrm{EUIG}$ and DyIG, resp. The magnetic moments are $(24 \pm 2) \mu_{B}$ per ficitive formula unit Eu3Fe 5012 and $(45 \pm 2) \mu_{B}$ for Dy $3 F_{5} 0_{12}$. The increase of moment below $90 \mathrm{~K}$ indicates ferrimagnetic order. No remanence is found down to $30 \mathrm{k}$. Even at $4.2 \mathrm{~K}$ a large amount of spins is unblocked. The magnetization in a field of $5 T$ is about half the value estimated for any callinear ferrimagnetic arrangement of the $\mathrm{Fe}^{3+}$, $\mathrm{Fe}^{2+}$ and $\mathrm{Eu}^{3+}$ resp. Dy $3+$ moments. The compensation temperature of nc DyIG is about $(35 \pm 2) \mathrm{k}$ but is strongly masked by the unblocked contribution to the magnetization.

\section{DISCUSSION}

The appearance of different valence states in sputter-deposited materials is well-known9. Here we can trace it to a preferential oxygen sputtering from the target: surface probes a 1 so contain $\mathrm{Fe}^{2+}$. During evaporation total molecular units may be conserved thus allow ing a close structural relationship between target and sputtered sample. We assume that the nc structure and its stoichiometry are stabilized by an oxygen deficiency $\delta$. Per formula unit RE ${ }_{3} F_{5} 0_{12-r}$ we estimated $\{\approx 0.4$ for nc EUIG and DyIG. These values compare with $\delta \approx$ $\approx 0.5$ reported for melt quenched nc GdIG10. The large efg for octahedral $\mathrm{Fe}^{3+}$ together with the increased isomer shift can be connected to a widening of the lattice with a corresponding distortion of the $02-$ octahedra6. The isomer shift of tetrahedral $\mathrm{Fe}^{3+}$ is enhanced in a similar way. The major part of the reduction of the saturation hf magnetic fields for $\mathrm{Fe}^{3+}$ can be explained by a distribution of the molecular field due to the dis-

Table I: ${ }^{57} \mathrm{Fe}$ hf parameters for non-crystalline (nc) and crystalline 4,5 (cr) EuIG and DyIG: isomer shift is rel. $\alpha \mathrm{Fe}(300 \mathrm{~K})$, quadrupole interaction $1 / 2 \mathrm{e}^{2} \mathrm{qQ}(300 \mathrm{~K})$, magnetic hf field Bhf( $4.2 \mathrm{~K})$, width $\Delta B h f / B h f$ of the Lorentzian hf field distributions, rel. occupation I for the different Fe sites, magnetic transition temperatures $T_{m}$.

EUIG

\begin{tabular}{|c|c|c|c|c|c|c|c|}
\hline & & $\mathrm{IS}_{300}(\mathrm{~mm} / \mathrm{s})$ & $\frac{1}{2} e^{2} q Q_{300}(\mathrm{~mm} / \mathrm{s})$ & $-B_{4.2}^{h f}(T)$ & $\Delta B^{h f} / B^{h f}$ & I & $T_{m}(K)$ \\
\hline & $\mathrm{Fe}^{3+}(\mathrm{tet} r)$ & 0.35 & 0.89 & 43.6 & \pm 0.05 & 2.55 & \\
\hline$n c$ & $\mathrm{Fe}^{3+}(\mathrm{oct})$ & 0.56 & 0.84 & 35.2 & \pm 0.2 & 1.70 & 62 \\
\hline & $\mathrm{Fe}^{2+}($ tet $r)$ & 0.68 & 1.84 & 27.1 & \pm 0.2 & 0.75 & \\
\hline & & \pm 0.01 & \pm 0.02 & \pm 0.5 & & \pm 0.05 & \\
\hline & $\mathrm{Fe}^{3+}(\mathrm{tet} r)$ & 0.21 & 0.85 & 47.5 & & 3 & \\
\hline$c r$ & $\mathrm{Fe}^{3+}(\mathrm{oct})$ & 0.46 & 0.45 & 54.5 & & 2 & 566 \\
\hline & $\mathrm{Fe}^{3+}(\mathrm{tet} r)$ & 0.38 & 0.90 & 45.1 & \pm 0.03 & 2.4 & \\
\hline$n c$ & $\mathrm{Fe}^{3+}(\mathrm{oct})$ & 0.56 & 0.84 & 39.0 & \pm 0.15 & 1.85 & 70 \\
\hline & $\mathrm{Fe}^{2+}(\mathrm{tetr})$ & 0.68 & 2.05 & 28.4 & \pm 0.4 & 0.75 & \\
\hline & & \pm 0.01 & \pm 0.02 & \pm 0.5 & & \pm 0.05 & \\
\hline & $\mathrm{Fe}^{3+}($ tet $r)$ & 0.20 & 0.90 & 48.0 & & 3 & \\
\hline & $\mathrm{Fe}^{3+}(\mathrm{oct})$ & 0.42 & 0.49 & 55.1 & & 2 & 563 \\
\hline
\end{tabular}


Eig. 3: Variation of the 57 Fe magnetic hf fields Bhf with temperature for tetrahedral $(x)$ and octahedral (e) $\mathrm{Fe}^{3+}$ and tetrahedral ( $\Delta) \mathrm{Fe}^{2+}$ in nc DyIG

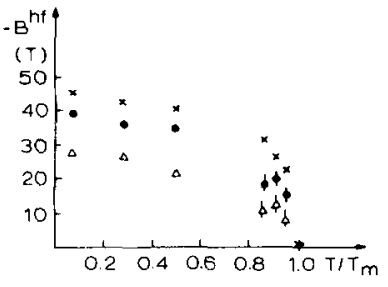

torted bonds in the nc solid which are responsible for the superchange. This distribution leads also to the observed distribution of $B^{\text {hf }}$. From this we calculate 11 a reduction of $4-6 \%$ for the hf field of tetrahedral $\mathrm{Fe}^{3+}$ and $18-24 \%$ for octahedral $\mathrm{Fe}^{3+}$. The larger distribution and thus the stronger reduction for octahedral $\mathrm{Fe}^{3+}$ are due to its higher number of bonds. The same arguments explain successfully the small saturation field of $\sim 28 \mathrm{~T}$ for tetrahedral $\mathrm{Fe}^{2+}$. In view of the relatively narrow distribution $\Delta B$ hf for tetrahedral $\mathrm{Fe}^{3+}$ it is not surprising that the variation of the $h f$ dield with temperature for this site is close to the functional dependence of the sublattice magnetization in a crystal. Unfortunately the data for the two other, are too inaccurate for a more quantitative analysis near $T_{m}$ where a less steep variation would be expected. The drastically reduced induced hf field at 151Eu3+ and also the high efg indicate a severe distortion of the RE dodecahedral sites accompanied by a reduction of the exchange field.

Previous? a strongly increased magnetic ordering temperature ( $\sim 850 \mathrm{~K}$ ) was reported for pyrolytic nc YIG 12 . In contrast to our results the Mössbauer data for this material are characterized by superparamagnetic effects. The agreement of the magnetic transition for nc EuIG and DyIG determined with $57 \mathrm{Fe}, 151 \mathrm{Eu}$ and $57 \mathrm{Fe}, 1610 y$, resp., with their different nuclear Larmor precession periods and the variation of the $57 \mathrm{Fe}$ hf fields near $T_{m}$ prove $T_{m}$ as true magnetic transition point, despite the fact that macroscopic magnetization is blocked at much lower temperatures. From the low magnetization at $4.2 \mathrm{~K}$ we propose a randomly canted spin arrangement although the overal1 order appears ferrimagnetic. Further Mössbauer epxeriments under an applied external magnetic field are needed to check whether the spin arrangement is really sperimagneticl 3 . The nearly tenfold reduction of $T \mathrm{~m}$ in comparison to the crystalline state cannot be explained by fluctuations of the Fe3+(tetr)$02--\mathrm{Fe}^{3+}$ (oct) superexchange alone. This would only result in a decrease of several percents. The hf field distribution gives no indication for the presence of nearly non-magnetic clusters. Thus percolation arguments 14 involving a simple non-magnetic dilution cannot be applied either. One possible mechanism is the unknown coupling of the tetrahedral $\mathrm{Fe}^{2+}$ to its neighbours. It could introduce some ferromagnetic $\mathrm{Fe}^{3+-\mathrm{Fe}^{2}+}$ double exchange. However, we propose that the dominant reason for the reduction of $T m$ lies in the reduction of the mean molecular field due to the widening of the lattice and the accompanying distortion of the bonds.

\section{CONCLUSION}

The microchemical composition and structure of sputtered nc EuIG and DyIG can be satisfactorily explained by a small oxygen deficiency due to preferential oxygen sputtering. Macroscopic magnetization suggests ferrimagnetic order possibly of sperimagnetic type. The sharp magnetic transition and the distribution of hf fields preclude a wide distribution of magnetic clusters with varying order parameters. Although a part of the deviations of the magnetic hf parameters from the values for the corresponding crystalline materials can be explained by a distribution in the molecular field, the strong reduction of $\mathrm{Tm}_{\mathrm{m}}$ must be attributed to a decrease of the average molecular field due to the distorted superexchange bonds. The present Mössbauer experiments will be completed by further $1610 y$ studies below $T_{m}$ on $n c$ DyIG in order to determine the sublattice magnetization of the rare-earth sites.

\section{REFERENCES}

a) Work supported by Bundesministerium für Forschung und Technologie

b) On leave from Universidad Barcelona, Spain

1. F.J. Litterst, G.M. Kalvius and A.J.F. Boyle, AIP Conf.Proc. 21, 616 (1975)

2. F.J. Litterst, J. Physique 36 L, 1977 (1975)

3. F.J. Litterst and G.M. Kalvius, Proc.Int. Conf. Mössbauer Spectroscopy Cracow 1975, pp189-220

4. I. Nowik and S. Ofer, Phys.Rev. 153, 409 (1967)

5. G. Crecelius, S. Hüfner and D. Quitmann, Proc.Conf.App 1. Mössbauer Effect, Tihany 1969, pp507-517

6. G. Amthaver, H. Amersten and S.S. Hafner, Z. Kristallogr. 143, 14 (1976)

7. B.D. Dunlap and J.G. Dash, Phys.Rev. 155, $460(1967)$

8. P. Kien1e, Rev.Mod.Phys. 36, 372 (1964)

9. H.M. Naguib and R. Kelly, Rad. Effects 25 , $1(1975)$

10. T. Matsuzawa, K. Okamura, T. Shishido and S. Yajima, J.Physique 40, C2, 149 (1979)

11. F.J. Litterst, V.D. Gorobchenko and G.M. Kalvius, J.Physique C, to be publ. 1979

12. Th.J.A. Popma and A.M. van Diepen, Mat. Res.Bul1.9, 1119 (1974)

13. J.M.D. Coey, J.Appl.Phys. 49, 1650 (1978)

14. A. Aharony, J.Magn. Magn. Mat. 7,198(1978) 